\title{
Comparison of synthetic jet actuators based on sharp-edged and round-edged nozzles
}

\author{
J. Kordík ${ }^{1, a}$ and Z. Trávníček ${ }^{1}$ \\ ${ }^{1}$ Institute of Thermomechanics, AS CR
}

\begin{abstract}
Axisymmetric synthetic jet actuators based on a loudspeaker and on two types of flanged nozzles were tested and compared experimentally. The first type of the nozzle was a sharp-edged circular hole. The second one had a special design with fillets at inner and outer nozzle exit and with a small step in the middle of the nozzle. The function of the step was to prevent the flow reattachment during the extrusion stroke. The actuators with the two types of nozzles were operated at resonance and were compared first qualitatively using a simple phase locked flow visualization. Then the hot-wire anemometer was used to measure velocity distributions along nozzle axis and velocity profiles at the nozzle exit. Comparison of the nozzles was based on evaluation of the characteristic velocity and integral quantities (volumetric, momentum, and kinetic energy fluxes). It was found out that these quantities, which were evaluated at the nozzle exit, differ substantially for both nozzles. On the other hand the velocity flow field in farther distances from the nozzle exit area did not exhibit such prominent differences.
\end{abstract}

\section{Introduction}

Synthetic jets (SJs) are fluid flows that are created by fluid pulsations and are formed in a free space behind a nozzle. The fluid is periodically pushed and pulled through the nozzle, whose one end exits into a cavity. The source of the pulsations is an oscillating diaphragm that bounds one side of the cavity. The nozzle is the only inlet/outlet in/from the cavity, therefore time mean mass flux through the nozzle is zero. It is the reason, why the SJs are sometimes called zeronet-mass-flux jets. The momentum flux along the nozzle axis is, however, non-zero. It causes the flow continuation ahead downstream from the nozzle exit and formation of a jet flow similar to the continuous jet.

Since the end of the last century, the topic of synthetic jets has been intensively investigated $[1,2]$ and many potential applications of SJs have been developed. The potential applications have been found especially in boundary-layer separation control [3-10], jet vectoring [11-16], heat transfer enhancement [17-22], and mixing [23-25]. A variant of the SJ, namely a non-zero-net-mass-flux jet (or hybrid SJ), has been investigated more recently [26-28,21,29-31].

If the synthetic jet actuators based on elastic diaphragms (e.g. based on a loudspeaker or a piezoceramic membrane etc.) and input/output energy balance are considered, then the most advantageous operating conditions are found at a resonance. Therefore, many authors concentrated their efforts on location and prediction of the SJ actuator resonances (see e.g. [32-44]). Having a SJ actuator operating at a resonance frequency, there still exist other ways how to enhance its exploitable outlet quantities such as volumetric flux, momentum or kinetic energy fluxes. This will be the

\footnotetext{
a e-mail: kordik@it.cas.cz
}

topic of this work. Particularly, this paper investigates the axisymmetric SJ actuators based on a loudspeaker and working at the first resonance. Under these conditions two types of flanged nozzles are tested within the actuator. The first type of the nozzle is a common sharp-edged circular hole and the second one possess fillets at inner and outer nozzle exit. The second nozzle was designed to have a lower hydraulic resistance and therefore, higher mentioned integral quantities (volumetric flux, momentum and kinetic energy flux) are anticipated. This paper is going to reveal whether this design is advantageous according to the expectations.

\section{Observed Parameters}

The first parameter, which is going to be observed and evaluated from the current experiments is the characteristic velocity of the SJ. This velocity is evaluated as follows:

$$
U_{0}=\frac{1}{T S_{\mathrm{n}}} \int_{0}^{T_{\mathrm{E}}} \int_{S_{\mathrm{n}}} u \mathrm{~d} S \mathrm{~d} t,
$$

where $S_{\mathrm{n}}$ is the nozzle exit cross-sectional area $(\mathrm{d} S$ is the differential element of $S_{\mathrm{n}}$ ), $u$ denotes the instantaneous velocity component aligned with the nozzle axis, $T_{\mathrm{E}}$ is the extrusion stroke duration time, $T$ is the actuation period, and the area-averaged velocity $\frac{1}{S_{\mathrm{n}}} \int_{S_{\mathrm{n}}} u \mathrm{~d} S$ has the origin at $t=0(t$ is time $)$.

A more simple evaluation of the SJ characteristic velocity can use a slug flow model approach, and the definition can be based on a point measurement of the centerline velocity waveforms only:

$$
U_{0, \mathrm{cl}}=\frac{1}{T} \int_{0}^{T_{\mathrm{E}}} u(r=0, x=0) \mathrm{d} S \mathrm{~d} t,
$$




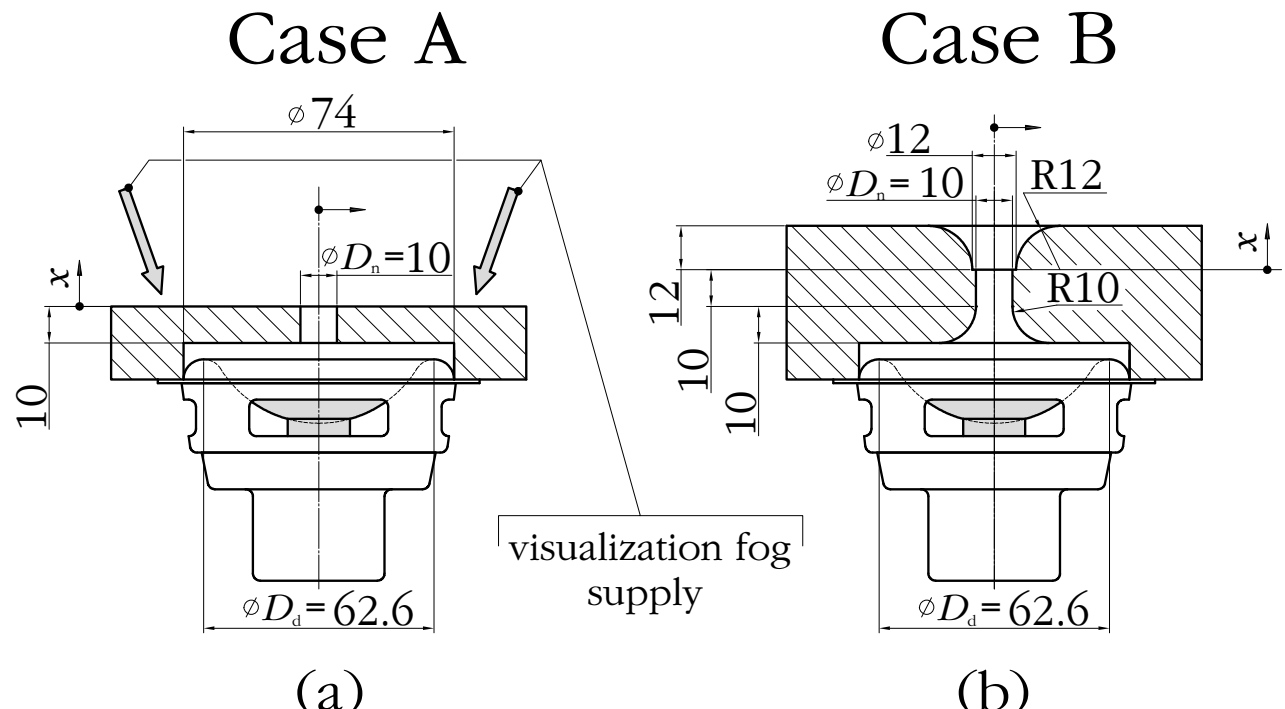

Fig. 1. Schema of the current actuators: (a) with a common, sharp-edged nozzle, (b) with a rounded nozzle.

Note that this relatively simple evaluation will be used for quantification of the velocity resonance curves - see paragraph 4.1 in the text below. The other parts of this study use more complex definition Eq. (1) which requires measurements of the velocity profiles across the jet.

The integral quantities, which are going to be analyzed, are: the time-average volumetric flux $\left(Q_{0}\right)$, momentum flux $\left(M_{0}\right)$, and kinetic energy flux $\left(E_{0}\right)$ :

$$
\begin{gathered}
Q_{0}=\frac{1}{T} \int_{0}^{T_{\mathrm{E}}} \int_{S_{\mathrm{n}}} u \mathrm{~d} S \mathrm{~d} t=U_{0} S_{\mathrm{n}}, \\
M_{0}=\frac{1}{T} \int_{0}^{T_{\mathrm{E}}} \int_{S_{\mathrm{n}}} \rho u|u| \mathrm{d} S \mathrm{~d} t, \\
E_{0}=\frac{1}{T} \int_{0}^{T_{\mathrm{E}}} \int_{S_{\mathrm{n}}} \frac{\rho u^{3}}{2} \mathrm{~d} S \mathrm{~d} t,
\end{gathered}
$$

where $\rho$ is the working fluid density.

Note, that the interval $\left(0, T_{\mathrm{E}}\right)$ can be a bit different for each quantity $Q_{0}, M_{0}$, and $E_{0}$. Generally, the interval represents time periods where the surface integrals from Eqs. $(3,4,5)$ are greater or equal to zero.

\section{Experimental Setup and Methods}

\subsection{The Synthetic Jet Actuators}

Figure 1 shows the schematic view of the tested SJAs that were operated at standard room conditions (temperature $20-25^{\circ} \mathrm{C}$ and barometric pressure $97-99 \mathrm{kPa}$ ) with air as the working fluid. The present actuators are based on a Aurasound NS3-193-8A loudspeaker, whose diaphragm generates the fluid oscillations in the nozzles. The main difference between the actuators dwells in their nozzle shapes. The first nozzle (case A) is a cylindrical hole - no special amendments lowering the hydraulic resistance are applied. The nozzle has a cylindrical shape and is sharp-edged at both flange surfaces. The second nozzle (case B) was designed with the aim to decrease the hydraulic resistance in both fluid flow directions. Therefore, the nozzle is rounded at its both surfaces. Moreover, as figure 1b shows, a small step in the middle of the nozzle that was designed to define flow separation from the step edge (i.e. to prevent the flow reattachment to the rounded wall) during the extrusion stroke. The narrowest cross-sectional area is the same for both tested nozzle shapes (the diameter is $D_{\mathrm{n}}=10 \mathrm{~mm}$ ).

During all experiments, the loudspeaker of the actuator was supplied by a harmonic electric current generated by Agilent 33220A function generator and gained in Pioneer A-209R amplifier. The experiments were performed at constant electrical power input into the actuator, $P_{\mathrm{e}}$. Three input power levels were used $P_{\mathrm{e}}=0.11,0.50$, and $0.54 \mathrm{~W}$.

\subsection{Data Acquisition and Hardware Setup}

To get a qualitative view on flow field patterns issuing from both tested nozzles (case A, case B), a simple flow visualization was conducted before quantitative hot-wire measurements. The visualization method used cold water fog produced by an ultrasonic piezoelectric nebulizer (Mini Nebler). The fog was blown at speed of $0.2 \mathrm{~m} / \mathrm{s}$ from the nebulizer using two supply pipes into the surroundings of the SJ orifice (fog supply spots are shown in figure 1a). The phase-locked SJ flow field patterns were observed under power-LED flashes synchronized with the actuator excitation frequency at an adjusted phase angle. Pictures were taken by a digital camera connected to a PC via an USB cable, using exposure time of $8.0 \mathrm{~s}$. The resulting photo is a multi-exposure of a large number of frames (typically 440 frames), which shows the phase-locked feature of the flow field and smoothing out of the deviation fluctuations in the individual cycles.

The MiniCTA 54T30 DANTEC hot-wire anemometer with a single wire probe 55P16 and the NI-PCI 6023E data acquisition device were used for this measurement. The hot-wire signal was sampled with a sampling frequency of 
$f \cdot 128$ and the number of samples was 25600 . The temperature of the working fluid (air) was measured using a fast-response Pt100 sensor (PT100-SMD0805) at the same time as the velocity samples. The measured temperature was used for the temperature correction of the hot-wire data. To evaluate and check the constant power input into the actuator, the supplied electric current and voltage were acquired concurrently with velocity and temperature samples.

After the identification of the velocity resonance frequency, the actuators were tuned to the resonance and velocity distributions along the $x$-axis were measured at power input of $P_{\mathrm{e}}=0.11 \mathrm{~W}$. The same experimental equipment and hardware setup as during auxiliary experiments was used.

At the same power level and frequency the velocity profiles were measured across the jet at the distances of $x=$ $0.3 \mathrm{~mm}, x=13 \mathrm{~mm}$, and $x=26 \mathrm{~mm}$. The velocity profiles measured at the distance $x=0.3 \mathrm{~mm}$ were acquired at 14 discrete positions along the nozzle diameter and the data were used for evaluation of $U_{0}$ and the integral quantities defined by Eqs. $(3,4,5)$.

For all measurements the hot-wire probe was calibrated in the velocity range of $0.15-62 \mathrm{~m} / \mathrm{s}$. The maximum relative uncertainty of a single velocity sample was below $5 \%$ for velocities in range of $0.4-62 \mathrm{~m} / \mathrm{s}$. For very small velocities in the range of $0.15-0.4 \mathrm{~m} / \mathrm{s}$ the uncertainty was $17.2 \%$. A more detailed description of the anemometer settings, probe calibration method, and uncertainty evaluation is available in [42].

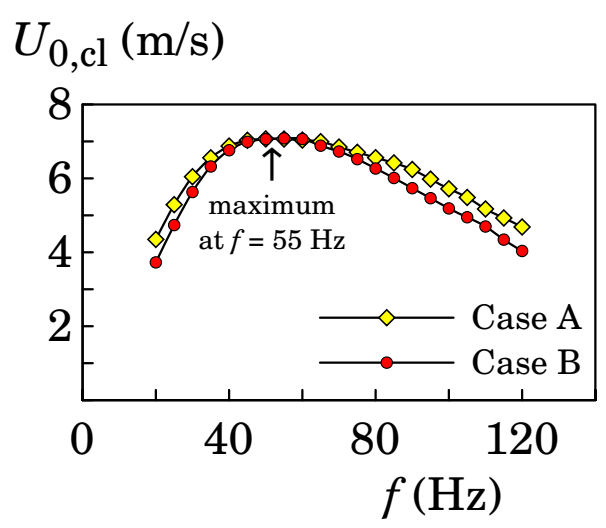

Fig. 2. Measured velocity resonance curves for both cases A and B.

\section{Experimental Results}

\subsection{Auxiliary Experiments}

The measured velocity resonance curves for identification of particular resonance frequencies are shown in figure 2. The two curves in figure 2 were acquired at constant power input into the actuator: $P_{\mathrm{e}}=0.5 \mathrm{~W}$ for both SJA cases $\mathrm{A}$ and $\mathrm{B}$. It was found out that the resonance frequency was the same for both nozzle types: $f=55 \mathrm{~Hz}$. We can extend this result also for other tested power levels, because as was shown in [31] the resonance frequency is nearly independent of the supplied power-input.

\subsection{Main Experiments and Discussion}

After identification of the resonance frequencies, SJs from both actuators (case A and B) were visualized by a water fog. The visualizations were performed at one power input level: $P_{\mathrm{e}}=0.11 \mathrm{~W}$ and at $f=55 \mathrm{~Hz}$ (the resonance frequency). The resultant pictures are shown in figure 3 for case A and in figure 4 for case B - see the photographs on left parts of the figures. Vortex formation process and its successive extinction can be observed in both figures. A phase shift of $\Delta \varphi \approx 30^{\circ}$ between visualizations in figure 3 and 4 is remarkable. This is caused by the additional top plate in case $\mathrm{B}$, which produces a spatial shift between the figures (the $x$-coordinate of the photographs in figure 4 starts at $\left.x / D_{\mathrm{n}}=1\right)$. Therefore, the initial vortex formation in the nozzle exit vicinity can not be observed. Apart from this phase shift there is no other remarkable qualitative difference between the visualized fluid flow patterns of both cases.

In the central column of Figs. 3 and 4 the distributions of instantaneous, $u$, and time-averaged velocities, $\bar{u}$, along the jet axis are shown. The traveling velocity maximum on these graphs roughly correspond with the vortex core position visualized by the water fog. Similarly as during the visualization, no distinct differences in the velocity distribution along the nozzle axis were found for both cases A and B.

The right parts of Figs. 3 and 4 show the velocity profiles measured at distances $x=0.3 \mathrm{~mm}, x=13 \mathrm{~mm}$, and $x=26 \mathrm{~mm}$. The measurements of the velocity profiles revealed several differences in the flow fields formed downstream from the both nozzle types. The velocity profiles measured at $x=0.3 \mathrm{~mm}$ are more flat during the extrusion stroke for the case B (rounded nozzle type), this will be important in evaluation of the integral quantities $Q_{0}, M_{0}$, and $E_{0}$, as it is shown below.

The velocity profiles measured at $x=13 \mathrm{~mm}$ are also very different. While in case A (plain nozzle) the suction phase of the actuator does not affect the velocity profile at this distance, negative velocities for $\varphi=180^{\circ}-360^{\circ}$ can be observed in case B. This is caused by the proximity of the additional wall with the rounded edge (see figure 1).

The differences between velocity profiles nearly disappear at the distance of $x=26 \mathrm{~mm}$. This can be also observed in Fig. 5 where the time-mean velocity profiles measured at $x=26 \mathrm{~mm}$ (i.e. $x / D_{\mathrm{n}}=2.6$ ) are displayed. The slight differences between the velocity profiles in figure 5 are within the measurement error, therefore, we can conclude that the profiles are practically identical.

The comparison of the integral quantities evaluated from the velocity profiles at the nozzle exit (more precisely at $x=0.3 \mathrm{~mm}$ ) offers the most prominent differences. The results are listed in table 1 . The nozzle associated with case B exhibited higher values in all observed parameters and quantities. The increase in $U_{0}$ and $Q_{0}$ was more than $18 \%$, even higher increase was found for $M_{0}$ 


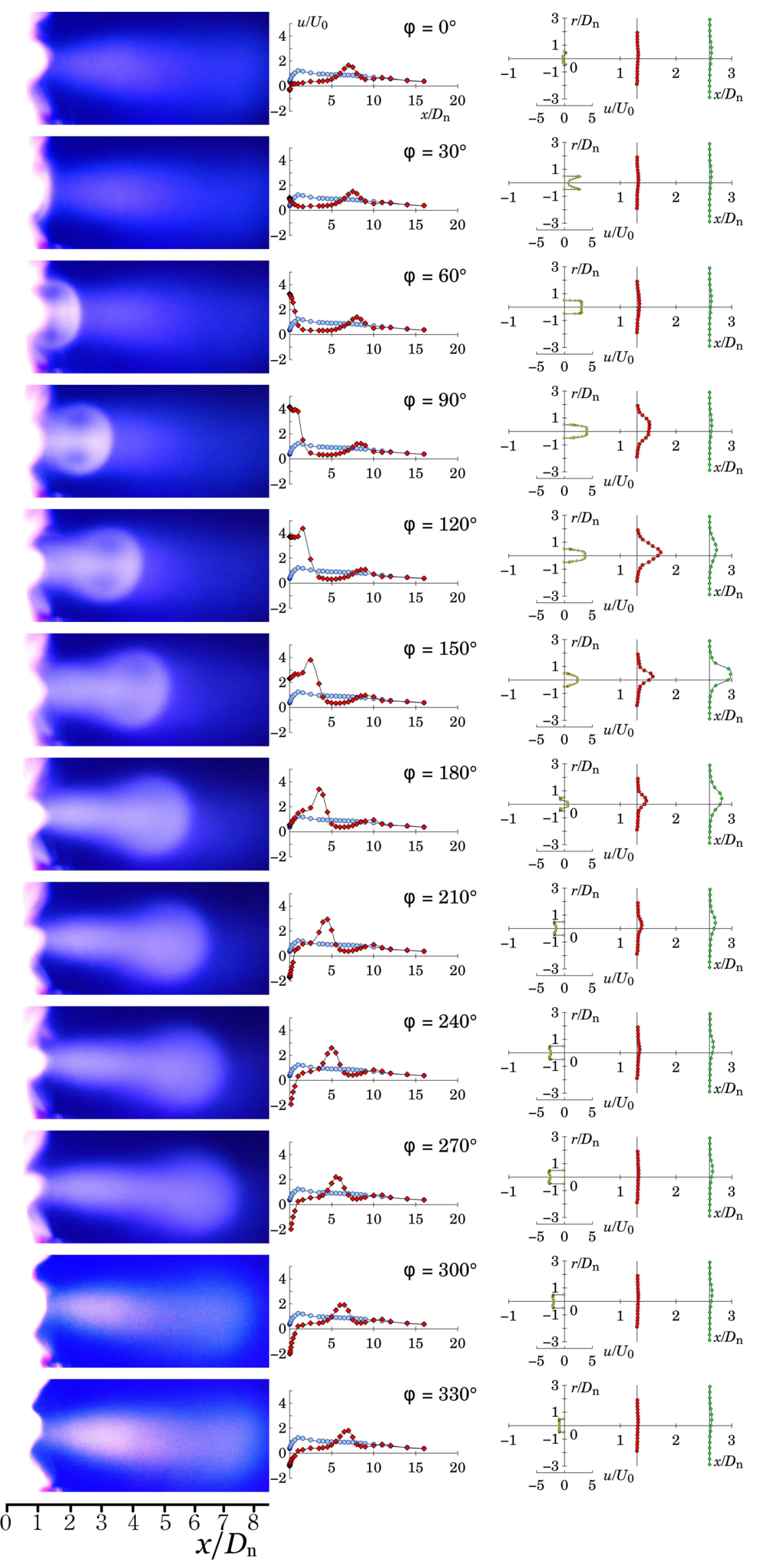

Fig. 3. Measurements results for case A, flow visualizations (left column), velocity distributions along the nozzle axis (central column), and velocity profiles (right column). 


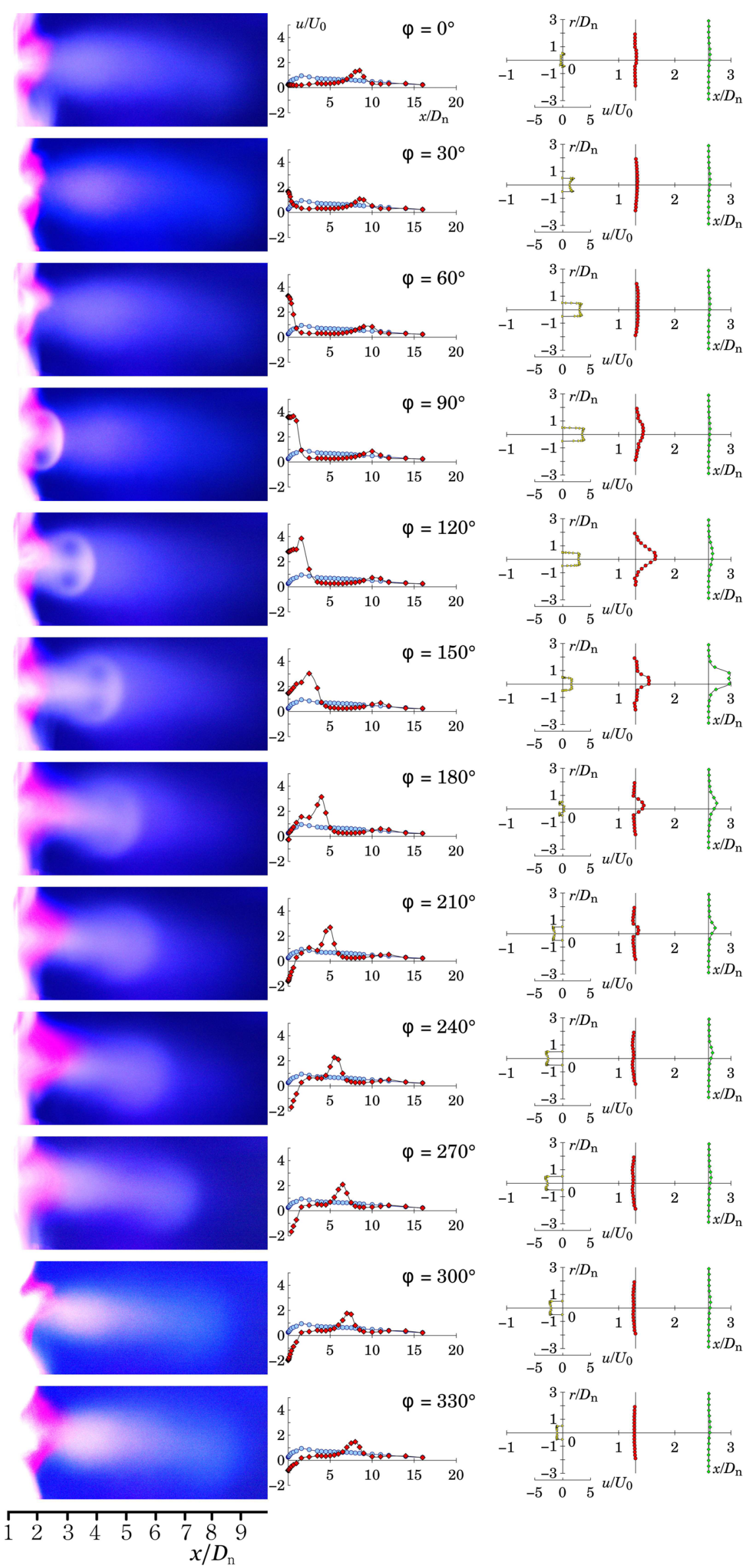

Fig. 4. Measurements results for case B, flow visualizations (left column), velocity distributions along the nozzle axis (central column), and velocity profiles (right column). 


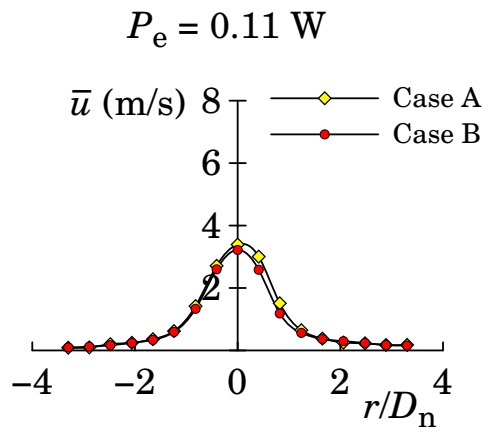

(a)

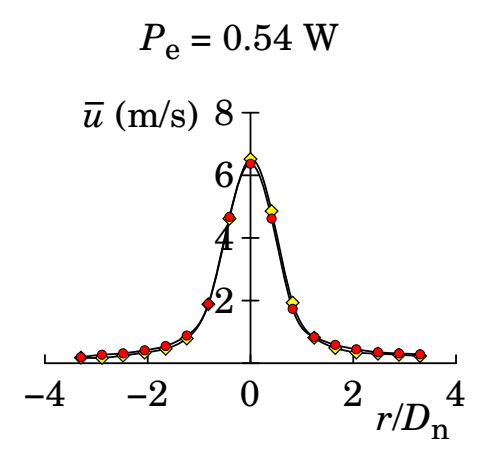

(b)

Fig. 5. Time-mean velocity profiles measured at the distance of $x / D_{\mathrm{n}}=2.6$ for input powers (a) $P_{\mathrm{e}}=0.11 \mathrm{~W}$ and (b) $P_{\mathrm{e}}=0.54 \mathrm{~W}$.

\begin{tabular}{rlccr} 
quantity & & case A & case B & increase \\
\hline$U_{0}(\mathrm{~m} / \mathrm{s})$ & $=$ & 3.4 & 4.1 & $18.5 \%$ \\
$Q_{0}(\mathrm{~m} 3 / \mathrm{s})$ & $=$ & $2.7 \times 10^{-4}$ & $3.2 \times 10^{-4}$ & $18.5 \%$ \\
$M_{0}(\mathrm{~N})$ & $=$ & $2.8 \times 10^{-3}$ & $4.0 \times 10^{-3}$ & $43.2 \%$ \\
$E_{0}(\mathrm{~W})$ & $=$ & $1.4 \times 10^{-2}$ & $2.4 \times 10^{-2}$ & $70.8 \%$
\end{tabular}

Table 1. The characteristic velocities and the integral quantities evaluated from the velocity profiles measured at $x=0.3 \mathrm{~mm}$ and for $P_{\mathrm{e}}=0.11 \mathrm{~W}$ using Eqs. $(1,3,4,5)$.

and $E_{0}: 43.2 \%$ and $70.8 \%$, respectively. This is a quite big difference, which shows the importance of evaluating these integral quantities from the velocity profile. For example, the velocities $U_{0}$ evaluated from the centerline waveforms at resonance - see e.g. figure 2 are practically identical, but the integral evaluation of $U_{0}$ shows the difference of $18.5 \%$.

Although the differences in the quantities from table 1 are large, figure 5 shows that differences between velocity profiles measured at $x / D_{\mathrm{n}}=0.26$ are negligible. Obviously, these differences cannot be explained without additional and more complex measurements in the near future.

\section{Conclusion}

Two types of flanged nozzles for a SJA were tested. The first type of the nozzle was a sharp-edged circular hole (case A) and second was the rounded nozzle (case B), which had a special design with fillets at inner and outer nozzle exit and with a small step in the middle of the nozzle (case B). The investigated actuator that was based on a speaker and worked at resonance and at a constant power input. Three input power levels were used: $P_{\mathrm{e}}=0.11 \mathrm{~W}, 0.5 \mathrm{~W}$, and $0.54 \mathrm{~W}$.

A phase locked flow visualization and hot-wire measurements were used for the qualitative and quantitative testing. Velocity profiles for the rounded nozzle (case B) and acquired at $x / D_{\mathrm{n}}=0.03$ were more flat during the extrusion stroke. Moreover, in case B, fluid suction influenced the velocity profile measured at $x / D_{\mathrm{n}}=1.3$, nothing similar was observed with the plain nozzle (case A).
The evaluation of the characteristic velocity and the integral quantities (volumetric flux, momentum flux, and kinetic energy flux) showed the most prominent differences between the nozzles. These quantities were evaluated from the velocity profiles measured at $x / D_{\mathrm{n}}=0.03$ and were always higher for the rounded type of the nozzle. A relative increase in the integral quantities was following: $18 \%$ (volumetric flux), $43.2 \%$ (momentum flux), and $70.8 \%$ (kinetic energy flux). On the other hand, the velocity profiles of the both tested cases A and B are nearly identical in the far field. To explain these facts, more complex measurements seem to be needed.

\section{Acknowledgement}

We gratefully acknowledge the support of the Grant Agency CR (project number 16-16596S) and the institutional support RVO:61388998.

\section{References}

1. B.L. Smith, A. Glezer, Physics of Fluids 10, 2281 (1998)

2. J.E. Cater, J. Soria, Journal of Fluid Mechanics 472, 167 (2002)

3. G. Hong, Sensor Actuat. A-Phys. 132, 607 (2006)

4. J. Dandois, E. Garnier, P. Sagaut, J. Fluid Mech. 574, 25 (2007)

5. D. You, P. Moin, J. Fluid Struct. 24, 1349 (2008)

6. S.G. Mallinson, J.A. Reizes, R. Hillier, Flow Turbulence and Combustion 66, 1 (2001)

7. M. Amitay, A. Glezer, International Journal of Heat and Fluid Flow 23, 690 (2002)

8. R. Rathnasingham, K.S. Breuer, Journal of Fluid Mechanics 495, 209 (2003)

9. S. Lardeau, M.A. Leschziner, Journal of Fluid Mechanics 683, 172 (2011)

10. C. Bernardini, M. Carnevale, M. Manna, F. Martelli, D. Simoni, P. Zunino, Journal of Thermal Science 21, 404 (2012)

11. B.L. Smith, A. Glezer, Journal of Fluid Mechanics 458, 1 (2002) 
12. Z.B. Luo, Z.X. Xia, Modern Physics Letters B 19, 1619 (2005)

13. Z.B. Luo, Z.X. Xia, Y.G. Xie, Chinese Journal of Aeronautics 20, 193 (2007)

14. Z.X. Xia, Z.B. Luo, Applied Mathematics and Mechanics 28, 907 (2007)

15. D.A. Tamburello, M. Amitay, International Journal of Heat and Fluid Flow 29, 967 (2008)

16. M. Ben Chiekh, M. Ferchichi, J.C. Béra, Experiments in Fluids 53, 1797 (2012)

17. M.B. Gillespie, M.S. thesis, Georgia Institute of Technology (1998)

18. Z. Trávníček, V. Tesař, International Journal of Heat and Mass Transfer 46, 3291 (2003)

19. M.B. Gillespie, W.Z. Black, C. Rinehart, A. Glezer, Journal of Heat Transfer 128, 990 (2006)

20. M. Arik, Applied Thermal Engineering 27, 1483 (2007)

21. Z. Trávníček, V. Tesař, J. Kordík, Journal of Visualization 11, 221 (2008)

22. M. Chaudhari, B. Puranik, A. Agrawal, International Journal of Heat and Mass Transfer 53, 1057 (2010)

23. H. Wang, S. Menon, AIAA Journal 39, 2308 (2001)

24. M. Al-Atabi, Journal of Fluids Engineering 133, 094503 (2011)

25. Q. Xia, S. Zhong, International Journal of Heat and Fluid Flow 37, 64 (2012)

26. Z. Trávníček, A.I. Fedorchenko, A.B. Wang, Sensors and Actuators A: Physical 120, 232 (2005)

27. Z. Trávníček, T. Vít, V. Tesař, Physics of Fluids 18, 081701 (2006)

28. V. Tesař, Sensors and Actuators A 138, 394 (2007)

29. J. Kordík, Z. Trávníček, Journal of Fluids Engineering 135, 101101 (2013)

30. Z. Trávníček, T. Vít, International Journal of Heat and Mass Transfer 85, 473 (2015)

31. J. Kordík, Z. Trávníček, M. Pavelka, Experimental Thermal and Fluid Science 69, 119 (2015)

32. Q. Gallas, M.S. thesis, University of Florida, Gainesville (2002)

33. Q. Gallas, J. Mathew, A. Kaysap, R. Holman, T. Nishida, B. Carroll, M. Sheplak, L. Cattafesta, Lumped element modeling of piezoelectric-driven synthetic jet actuators, in 40th AIAA Aerospace Sciences Meeting $\mathcal{G}$ Exhibit (AIAA Paper 2002-0125, Reno, 2002)

34. Q. Gallas, R. Holman, T. Nishida, B. Carroll, M. Sheplak, L. Cattafesta, AIAA Journal 41, 240 (2003)

35. Q. Gallas, M. Sheplak, L. Cattafesta, Final report for NAG-1-03031, University of Florida, Department of Mechanical and Aerospace Engineering, Gainesville (2005)

36. Q. Gallas, R. Holman, R. Raju, R. Mittal, M. Sheplak, L. Cattafesta, Low dimensional modeling of zero-net mass flux actuators, in 2nd AIAA Flow Control Conference (AIAA Paper 2004-2413, Portland, 2004)

37. Q. Gallas, PhD. thesis, University of Florida, Gainesville (2005)

38. B.H. Kim, D.R. Williams, S. Emo, M. Acharya, AIAA Journal 43, 314 (2005)
39. H. Tang, S. Zhong, Lumped element modelling of synthetic jet actuators, in The 3rd AIAA Flow Control Conference (AIAA Paper 2006-3696, San Francisco, 2006)

40. H. Tang, S. Zhong, M. Jabbal, L. Garcillan, F. Guo, N. Wood, C. Warsop, Flow, Turbulence and Combustion 78, 309 (2007)

41. H. Tang, S. Zhong, Aerospace Science and Technology 13, 331 (2009)

42. J. Kordík, Synthetic and Hybrid Synthetic Jet Actutators - Theoretical and Experimental Analysis (LAP LAMBERT Academic Publishing, 2013), ISBN 978-3659-32910-4

43. J. Kordík, Z. Trávníček, AIAA Journal 51, 2862 (2013)

44. J. Kordík, Z. Broučková, T. Vít, M. Pavelka, Z. Trávníček, Experiments in Fluids 55, 1757 (2014) 